\title{
Investigation on the Temporal Surface Thermal Conditions for Thermal Comfort Researches Inside A Vehicle Cabin Under Summer Season Climate
}

\author{
Wencan Zhang ${ }^{1, a}$, Jun Liu ${ }^{1}$ \\ ${ }^{1}$ School of Mechanical \& Electrical Engineering, Foshan University, Foshan 528000, China
}

\begin{abstract}
With the proposes of improving occupant's thermal comfort and reducing the air conditioning power consumption, the present research carried out a comprehensive study on the surface thermal conductions and their influence parameters. A numerical model was built considering the transient conduction, convective and radiation heat transfer inside a vehicle cabin. For more accurate simulation of the radiation heat transfer behaviors, the radiation was considered into two spectral bands (short wave and long wave radiation), and the solar radiation was calculated by two solar fluxes (beam and diffuse solar radiation). An experiment was conducted to validate the numerical approach, showing a good agreement with the surface temperature. The surface thermal conditions were numerically simulated. The results show that the solar radiation is the most important factor in determining the internal surface thermal conditions. Effects of the window glass properties and the car body surface conditions were investigated. The numerical calculation results indicate that reducing the transitivity of window glass can effectively reduce the internal surface temperature. And the reflectivity of the vehicle cabin also has an important influence on the surface temperature, however, it's not so obvious as comparison to the window glass.
\end{abstract}

\section{Introduction}

Thermal comfort in vehicles cabins is one of the major concerns by car's owners, researchers and automotive industries as it is known to affect the occupant's travel experience as well as the driver's physiological arousal activity level and concentration ability. It has now become an important criterion for consumers to select a new car. Some researches indicate that the principal factors affecting the occupant's thermal comfort depend on the such thermal conditions as air temperature, relative humidity, mean radiant temperature, and air velocity; in addition to the clothing worn on the occupant and the physical activity of the occupant[1]. Most of investigations on occupant's thermal comfort focus on the thermal environment, especially of the air-flow field and the temperature distributions during cooling or heating period in vehicle cabin[2-6]. The surface temperature plays an important role. The thermal conditions of the surface which contact with occupant segment, for example the seat and the steering wheel, have a direct impact on the occupant's thermal comfort. Moreover, low or high surface temperature surrounding the occupant will lead to an unwanted mean radiant temperature. On a hot summer day, the internal surface temperature of a vehicle parked in the

\footnotetext{
${ }^{\text {a } C o r r e s p o n d i n g ~ a u t h o r ~: ~ z h a n g w e n c a n @ l i v e . c o m ~}$
} 
sun can exceed $90^{\circ} \mathrm{C}$, it greatly affect the occupant's thermal comfort. Reducing the temperature of the vehicle interior surface can greatly improve passenger and driver's travel experience. However, few studies have been undertaken focusing on the surface conditions in a vehicle cabin.

Today, with the urgent need to reduce the economic and environmental cost of energy consumption, not only thermal comfort but also the energy efficiency has been recognized as essential parameters of vehicle cabin environmental design. Air conditioning is the major ancillary load for a compact vehicle. As a quantitative example, approximately 26 billion liters of fuel is consumed for the HVAC system in vehicle cabin annually in the United States[7]. The HVAC system in vehicle cabin wastes a large amount of energy, which is used to cool the entire vehicle cabin rather than just occupants. In a cooling period, a person can produce only about $100 \mathrm{~W}$ of heat. However, a typical cooling system in a vehicle provides about $4000 \mathrm{~W}$ of cooling[8]. The study of Levinson et al shows that most of the heat removed by the air conditioning comes from the cabin surface, rather than the air[9]. Many studies have considered the vehicle cabin as a potential approach to reduce ancillary load and improve fuel economy by decrease the its surface temperature. Turler al et[10] experimental studied the effect of using advanced thermal insulation material and window technologies on reducing vehicle auxiliary loads, the test results indicated that the retrofitted vehicle warm up under soak conditions was delayed compared to a factory vehicle. In Hoke and Greiner's study[11], experiments and numerical simulations were conducted to determine the effect of paint properties on the thermal load present and the resulting temperatures during vehicle soak. They found out that decreasing the solar absorptivity of the painted surface reduced the vehicle soak temperature and the transient air conditioning cool down time to comfort. Levinson al et[9] performed an experimental comparison of otherwise identical black and silver compact sedans, the results indicated that increasing the solar reflectance of the car's body by about 0.5 lowered the soak temperature of breath level air by about 5$6^{\circ} \mathrm{C}$. And the air conditioning capacity required to cool the cabin air in the silver car to $25^{\circ} \mathrm{C}$ within 30 $\min$ is $13 \%$ less than that required in the black car.

This current study focuses on the internal surface thermal conditions of a vehicle cabin under the sun. Firstly, a description of the heat transfer modes of a vehicle at daytime and the theoretical support of the simulation is presented. Secondly, an experiment is conducted under the typical climate conditions of South China summer season with the propose to validate the calculation model. Then, the numerical simulations are performed to investigate the effect of the vehicle window and body properties on the interior surface temperature. And this paper ends with a summary of major conclusions.

\section{Numerical method}

Fig. 1 shows the basic heat transfer modes of a vehicle at daytime. As shown, the heat transfer modes include conduction, convection and radiation. The short wave radiation can penetrate the glass window and goes into the cabin but the long wave radiation emitted by the cabin internal surface is prevented from escaping. Thus, in order to propose an accurate and feasible vehicle cabin heat transfer mode, the radiations considered here are divided into the long wave radiation and the short wave radiation. The short wave radiation includes the solar radiation; while the long wave radiation includes the sky radiation, the environment radiation and the surface radiation. The solar radiation can be calculated by two fluxes: the direct solar radiation and the diffuse solar radiation.

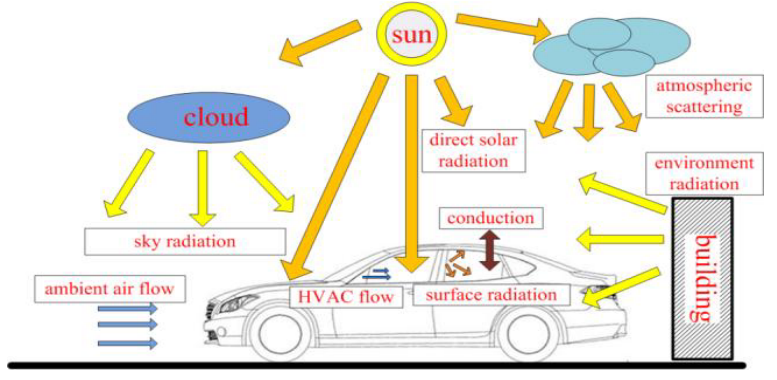


Figure 1. Heat transfer process of vehicle

The nodal network meth was used for numerical calculation, the procedure is shown as Fig. 2: (a) discretize the vehicle cabin into a series of small elements from an original CAD model, and set up the material properties for the vehicle cabin; (b) calculate the view factor; (c) obtain and set up the initial conditions; (d) calculate the heat transfer of the vehicle cabin, obtain the surface and air temperatures; (e) validate the numerical results with experiment data; (f) according to the validation result, repeat (a) to (e), or accept the numerical results.

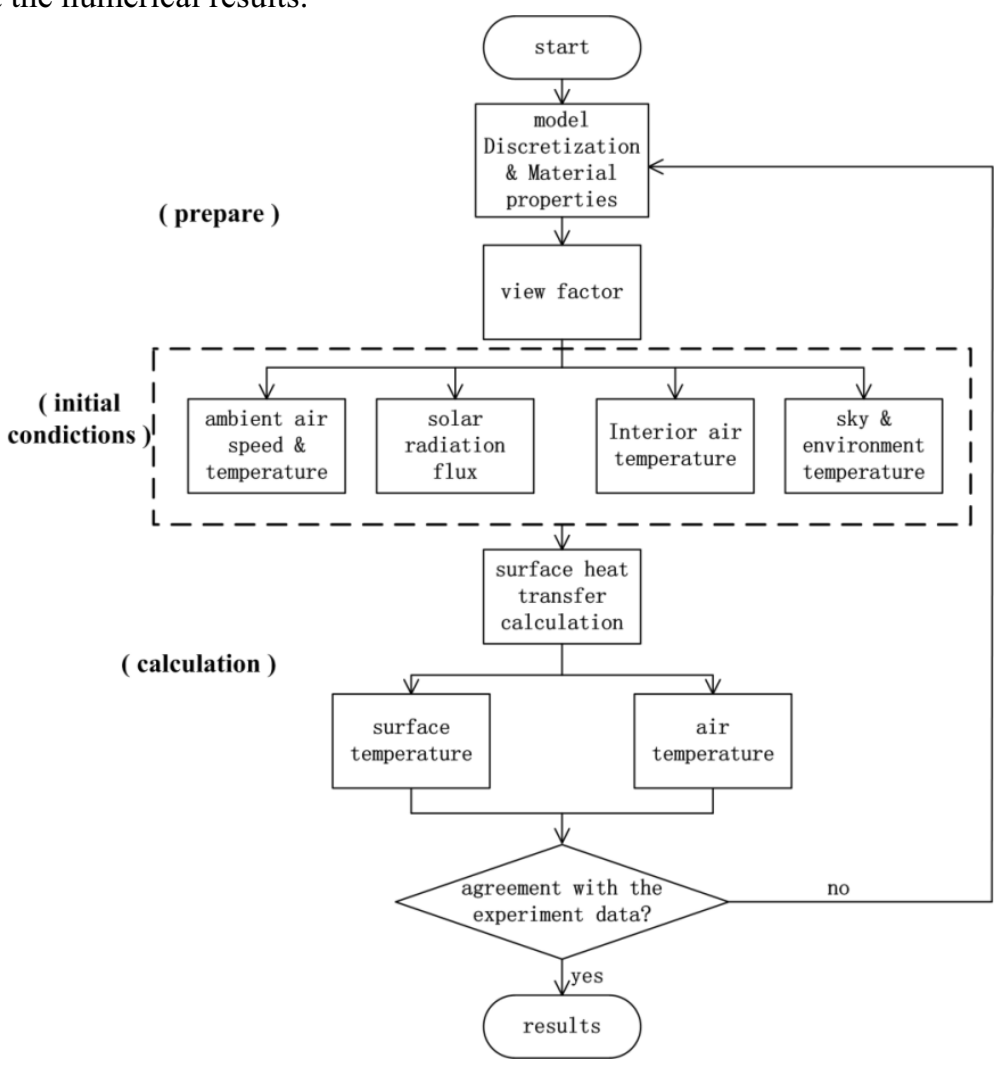

Figure 2. The numerical solution procedure

\section{Experiment}

For the purpose of validating the heat transfer calculation methods and the numerical model, an experiment was designed and conducted at the State Key Laboratory of Environment Adaptability for Industrial products in the summer season climate of south China. As shown in Fig. 3(a), a sedan parked in the outdoor turf with direct exposuring to sunlight was used in the test. The test vehicle was heated by different solar radiation intensity in the daytime. A small meteorological station shown in Fig. 3(b) was applied to obtain the meteorological conditions. According to the aforementioned heat transfer process of vehicle, the environment conditions including the ambient temperature, the ambient air speed, the direct solar radiation and the diffuse solar radiation flux were monitored during the experiment. The internal surface temperature and the interior air temperature were collected by using a discrete thermocouples network. The surface temperature at the dashboard, the seats and the doors were monitored, these places are expected to have a significant influence on the occupant's thermal comfort. Fig. 3(c) is an illustration of the surface temperature measurement method in the present study. The thermocouple was adhered to the surface by a type of light color material with low radiation absorptance to minimize the absorption of the solar radiation and surface radiation which 
would lead to an over measurement in surface temperature. Another thermocouple suspended approximately $150 \mathrm{~mm}$ from the ceiling in the middle of the cabin was used to measure the interior air temperature. In order to avoid exposing to direct sunlight, the thermocouple was wrapped in a small cylinder with low solar absorptance and low thermal emittance as shown in Fig. 3(d).

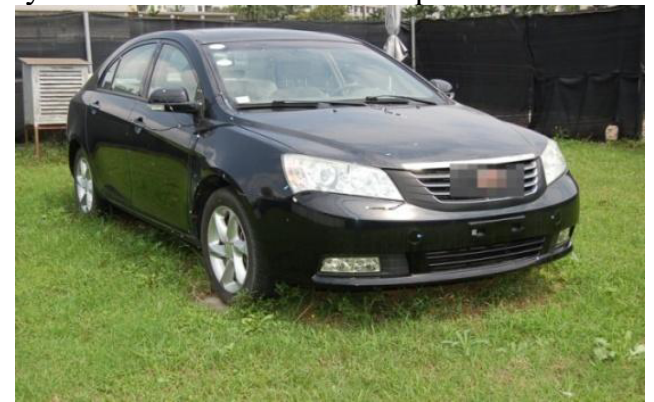

(a)

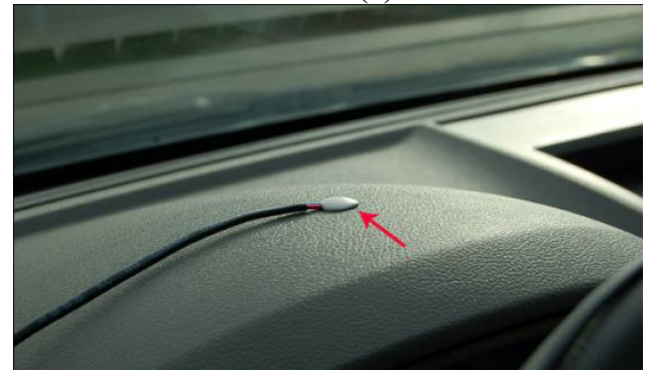

(c)

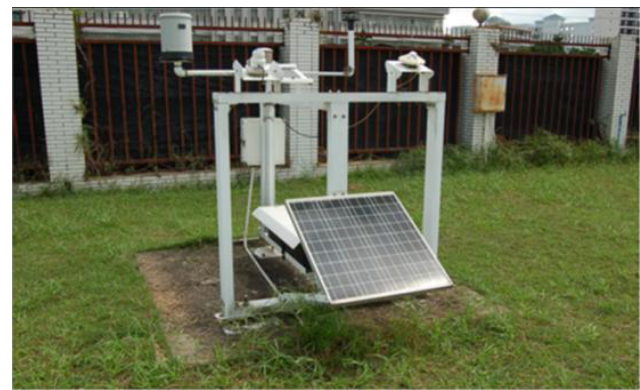

(b)

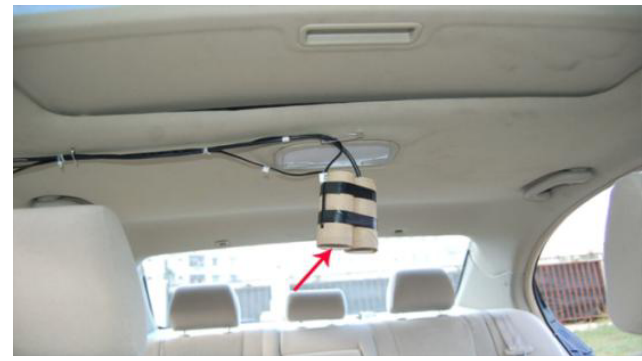

(d)

Figure 3. Test vehicle, Meteorological station, surface and air temperature measurement in the test

\section{Results and discussion}

\subsection{Experiment results}

The test was carried out from 6:00 to 18:00. The temperature measurement data were collected every 30 seconds by using a data acquisition system integrates with and a laptop computer, while the meteorological conditions were measured in 10 minutes intervals during the test. Fig. 4 gives the radiation flux and temperature measurement results in the experiment. As shown, the diurnal temperature variation is greatly depended on the solar radiation. The peak temperature at each surface always occurs between 12:00 to 14:00 when the solar has its maximum irradiation intensity. During the soaking period, the temperature distribution inside the vehicle cabin is highly non-uniform, the temperature differences among these surfaces can exceed $30^{\circ} \mathrm{C}$. Because the sun rises from the east in the summer season, the east-facing surfaces in the test usually have higher temperature. The hottest surface in the present experiment is the dashboard due to it is completely exposed to the solar radiation transmitted through the windshield, its peak temperature is about $90^{\circ} \mathrm{C}$, the next hottest surfaces are the seats, which also have large area exposed to the solar radiation. Another observation is that a greenhouse effect presents in the vehicle cabin. The temperature profiles of both interior and outside ambient air are similar. However, the interior air temperature is well above the outside ambient temperature. It is because the solar radiation can pass through the glass window of the vehicle and heats the internal surfaces, while the long wave radiation emitted by the internal surfaces is not able to pass through the window. Thus, all the internal surfaces will reach a considerable high temperature. Then, the interior air is conductively and convectively heated by the hot internal surface. Under the influence of this greenhouse effect, the interior air temperature is up to $60^{\circ} \mathrm{C}$ and about $30^{\circ} \mathrm{C}$ higher than that of the outside ambient air. 

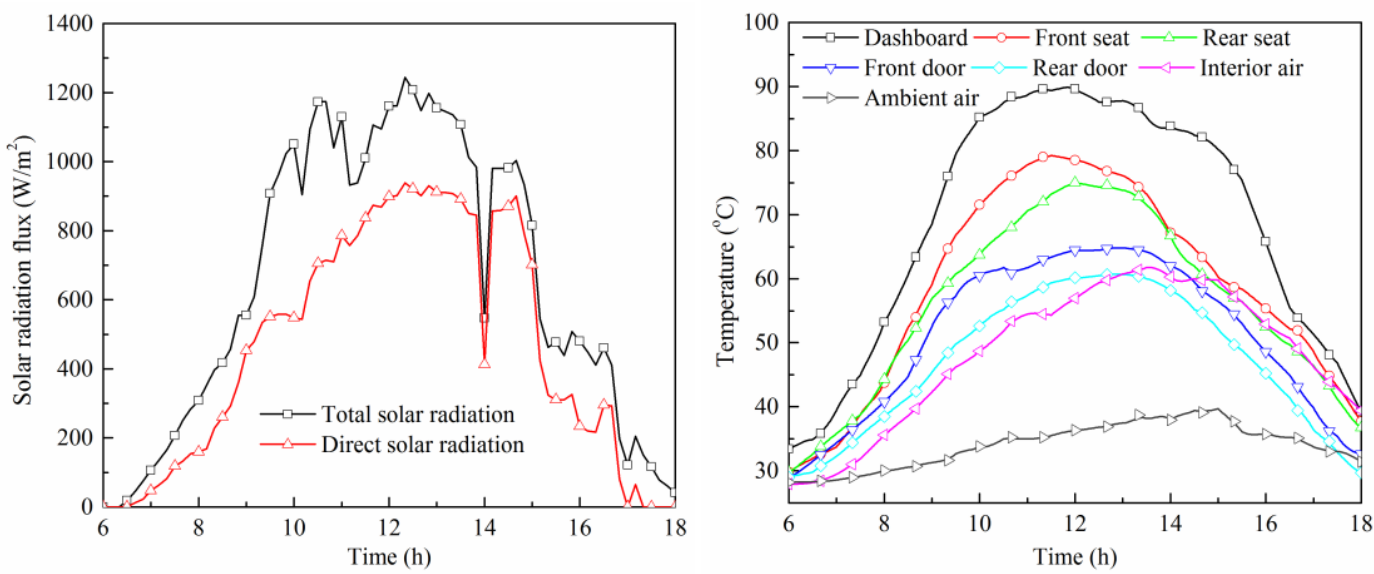

(a)

(b)

Figure 4. Experiment results: (a) the solar radiation flux; (b) temperature

\subsection{Numerical model validation}

The experiment results demonstrated above are use to validate the numerical model and methods. Table 1 lists the material properties of the vehicle cabin used in the simulation model.

Table 1. Material properties of the vehicle cabin used in the simulation model

\begin{tabular}{ccccc}
\hline Parts & Material & Density $\left(\mathrm{kg} / \mathrm{m}^{3}\right)$ & Specific heat $(\mathrm{J} / \mathrm{kg} \cdot \mathrm{K})$ & Thermal conductivity $(\mathrm{W} / \mathrm{m} \cdot \mathrm{K})$ \\
\hline Car body & Steel & 7785 & 449 & 100 \\
Door & ABS & 996 & 1464 & 0.166 \\
Dashboard & Lexan & 1200 & 1502 & 0.3 \\
Seat & Leather & 860 & 1500 & 0.135 \\
Window & Glass & 2530 & 754 & 1.1717 \\
\hline
\end{tabular}

Fig. 5 shows the comparisons between the simulation temperature profiles and the experiment data for both internal surfaces of the vehicle cabin. It can be observed that the simulation results are in good agreement with the values of experiment for all the internal surfaces temperature. The maximum surface temperature deviation between the predicted and the measured result is about $5{ }^{\circ} \mathrm{C}$ and within $10 \%$. Therefore, this simulation method is able to satisfactorily predict the internal surface temperature variation of the vehicle cabin under the sun.

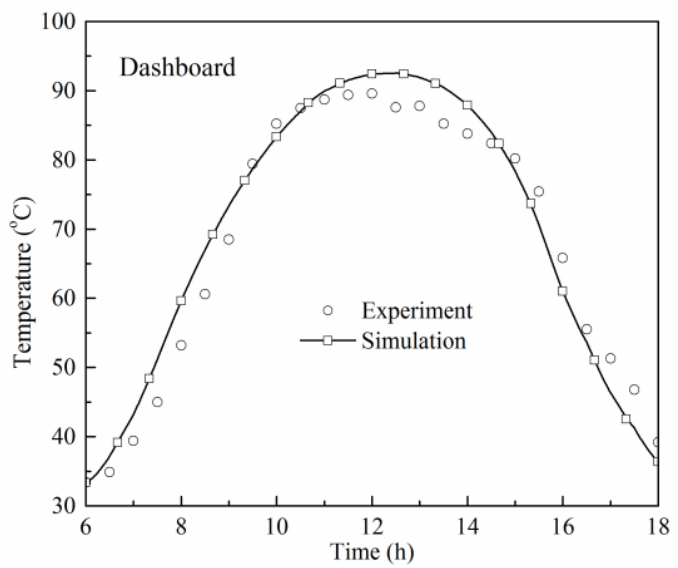

(a)

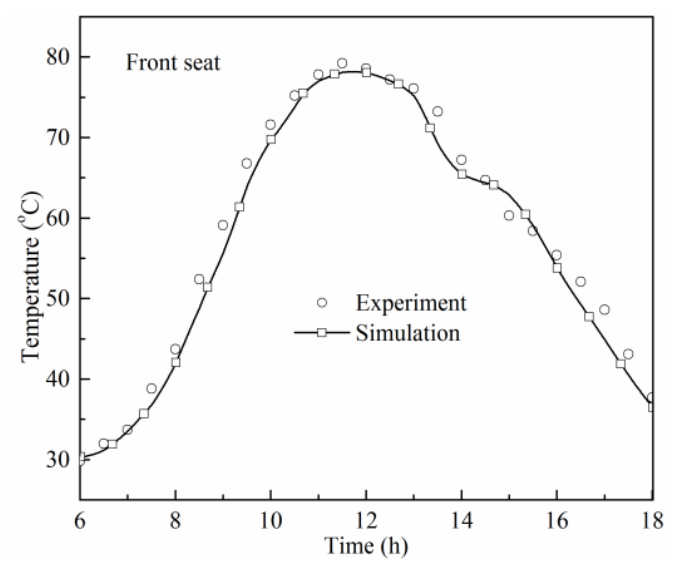

(b)

Figure 5. Comparisons between the simulation and experiment temporal temperature variation: (a) Dashboard; (b) Front seat 
To investigate the contribution of each type heat transfer mode to the internal surface temperature, Fig. 6 gives the net heat flux of the conduction, convection, surface radiation and solar radiation on each surface. As shown in Fig. 6(a)-(c), the solar radiation received by the dashboard and the seats is very large. It is why the dashboard and the seats have high surface temperature when soaking in the sun as described above. And their heat dissipates conductively, radiatively and convectively to other surfaces and to the air. The heat flux evolution of the seats is not smooth as depicted in Fig. 6(b) and (c), a shake-up is found in the solar radiation heat flux profile. It is due to the shade effect of the car body and the internal surfaces, the solar radiation heat flux sharp increases when the solar irradiation reaches the surface and sharp decreases when the solar irradiation is shaded by the car body and other surfaces. Fig. 6(d) shows the net heat flux of the doors, unlike the dashboard and the seats, the solar radiation heat flux is very small. The influence of the solar radiation to their surface temperature is not the most important as compares to other types of heat transfer. Another important phenomenon revealed in Fig. 6(d) need to be pointed out is the net conduction heat flux. During the soaking period, there is dynamic temperature difference between the internal surface and the external surface of the door. At the first stage, the external surface temperature is higher than that of the internal surface due to it is exposed to the sun illumination and receives more solar radiation, the internal surface of the door is conductively heat by the external surface. At the second stage, the external door surface temperature is lower than that of the internal surface. Because the solar radiation received by the external surface decreases due to the position of the sun has changed and its radiation intensity weakens. Furthermore the external surface is also cooled convectively by the ambient air. Thus, the internal door surface is cooled conductively by the external surface, and its net conduction heat flux decreases and becomes negative.

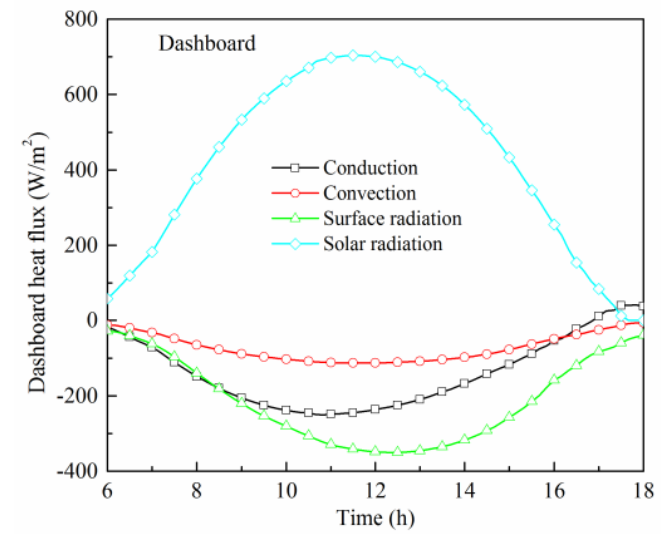

(a)

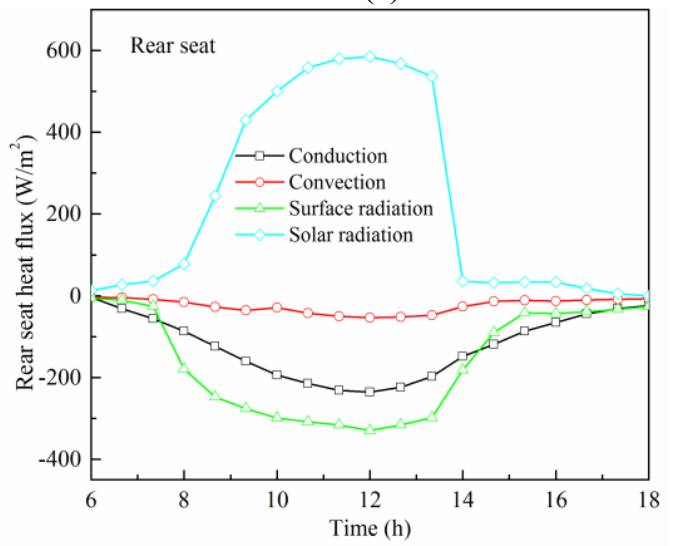

(c)

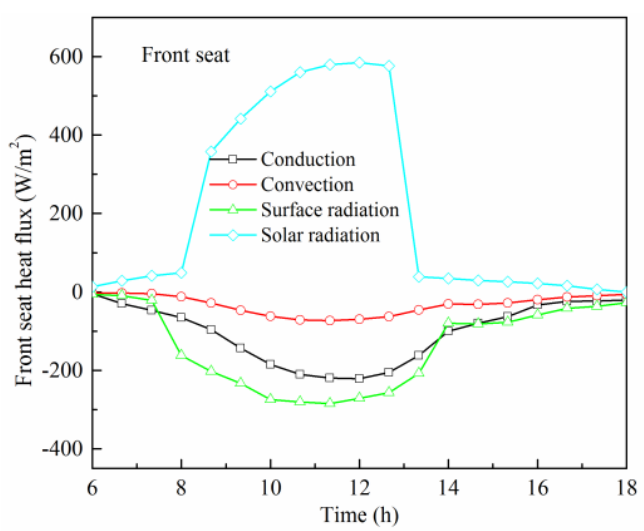

(b)

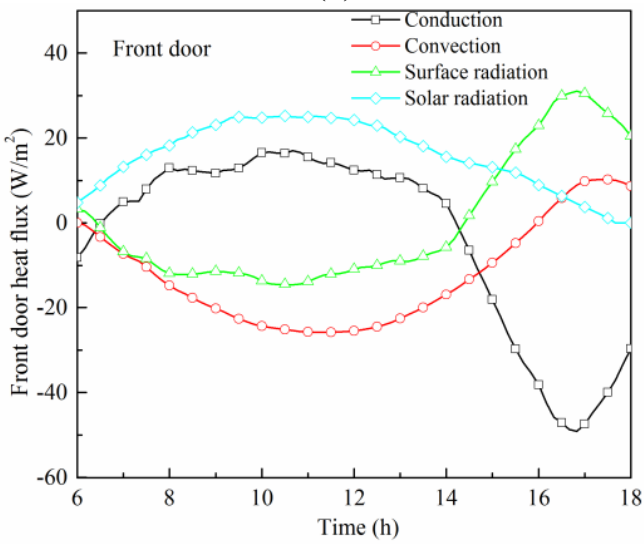

(d)

Figure 6.The heat flux of each internal surface 


\subsection{The effect of window glass properties}

As aforementioned, the solar radiation has a decisive influence on the internal surface temperature of a vehicle cabin. Restricting the solar irradiance into the cabin is an effective method to reduce the surface temperature, then to improve the occupant's thermal comfort and reduce the energy consumption of the HVAC system in the cabin. 4 different types of glasses are selected for parametric analysis, in order to quantify the effect that the glass window on the internal surface temperature distribution. The glass optical properties, including the base line and other three types, are given as table 2 .

Table 2. Optical properties of the four glass types

\begin{tabular}{llll}
\hline Glass type & Transmittivity & Absorptivity & Reflectivity \\
\hline Base line & 0.76 & 0.16 & 0.08 \\
Glass type I & 0.56 & 0.16 & 0.28 \\
Glass type II & 0.36 & 0.16 & 0.48 \\
Glass type III & 0.16 & 0.16 & 0.68 \\
\hline
\end{tabular}

Fig. 7 depict how the window glass can affect the internal surface temperature distribution of a vehicle cabin. As can be seen, the glass optical properties have a pronounced influence on the temperature of all the surfaces. However, the sensitivity of different surfaces to the optical properties is quite different. As previously noted, the dashboard is just behind the front windshield and it is totally exposed to the solar illumination, its surface temperature is greatly depended on the solar intensity passed through the windshield. When decrease the transmittivity of the glass, the solar radiation energy obtained by the dashboard is significantly reduced. Therefore, the dashboard is the most sensitivity surface to the glass optical properties. From the geometric of the vehicle cabin and the location of the sun, it's obvious that the next sensitivity surfaces are the seats and then the doors. As revealed in Fig. 7(d), the maximum surface temperature at the dashboard and the seats greatly reduce with the transmittivity of window glass reduces (reflectivity of the glass increases accordingly). When the transmittivity of window glass reduces each 0.2 , the maximum surface temperature reduces about $15^{\circ} \mathrm{C}$ and $9^{\circ} \mathrm{C}$ at the dashboard and the seats correspondingly. As above mentioned, the solar radiation received by the internal door surface is very small and its impact is not the most important as comparison to other factors. Therefore, reducing the transmittivity of window glass will not have significant influence on the internal door surface, it only reduces about $2^{\circ} \mathrm{C}$ when the transmittivity of window glass reduces each 0.2 .

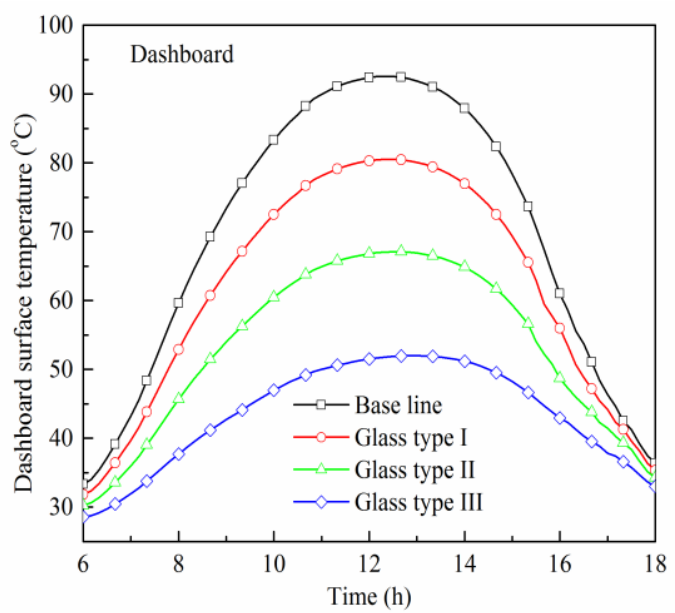

(a)

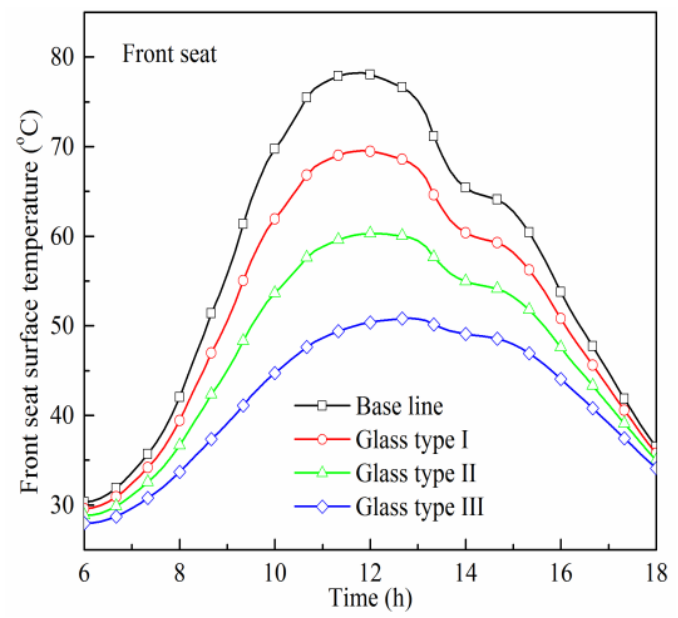

(b) 


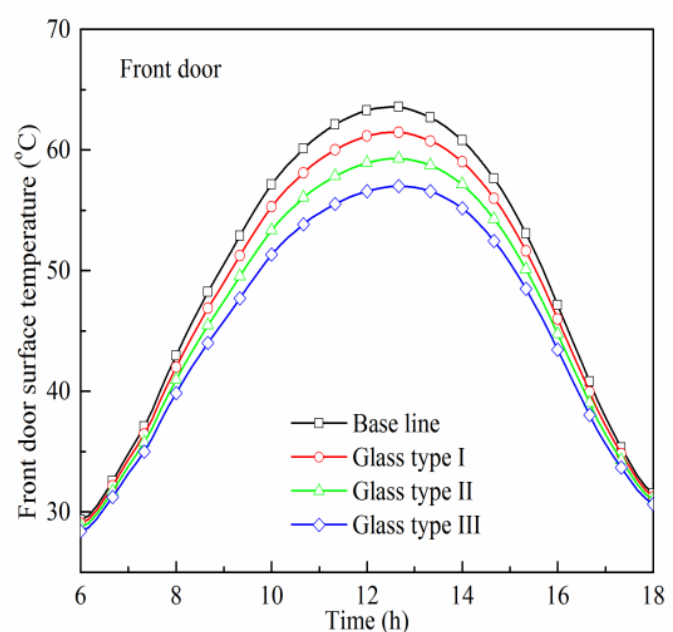

(c)

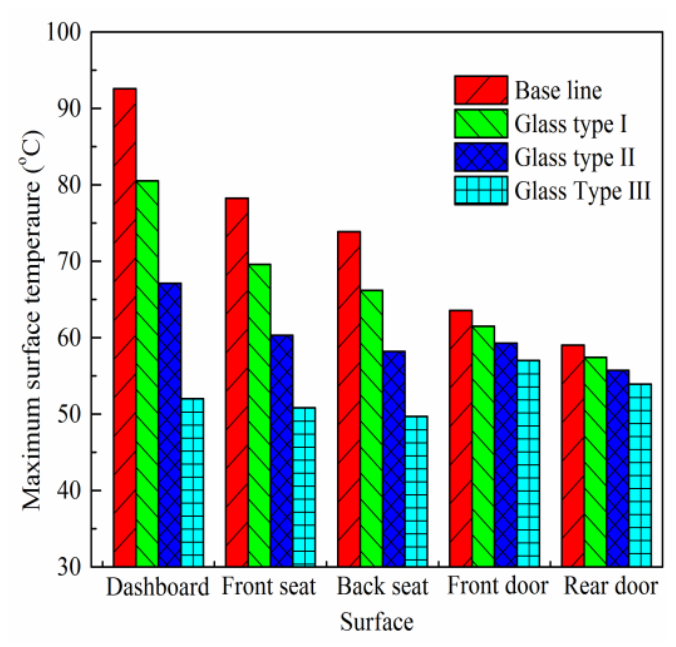

(d)

Figure 7. Effect of glass properties on the internal surface temperature

\subsection{The effect of car body surface conditions}

The car body surface condition is another major parameter that affects the vehicle cabin interior thermal environment, changing the car body surface condition is also an especially effective process to reduce the cabin surface temperature, and then the interior air temperature and the fuel consumption. To quantify the influence of the external surface absorptivity and reflectivity on the internal surface temperature, 4 types of surface conditions listed in table 4 are considered in this study.

Table 3. surface conditions of the four car body types

\begin{tabular}{lll}
\hline Surface condition & Absorptivity & Reflectivity \\
\hline Base line & 0.74 & 0.26 \\
Surface condition I & 0.54 & 0.46 \\
Surface condition II & 0.34 & 0.66 \\
Surface condition III & 0.14 & 0.86 \\
\hline
\end{tabular}

As expected, decreasing the absorptivity or increasing the reflectivity of the external surface results in a decrease of internal surface temperature of the vehicle cabin as shown in Fig. 8. As depicted, the effect of car body surface condition is not so obvious as comparison to the properties of window glass. From Fig. 8(d), it is known that the most sensitive surface to the external surface condition is the door. Once decreases the absorptivity by each 0.2 (increase the reflectivity by 0.2 accordingly), the maximum temperature of the door would decrease about $4^{\circ} \mathrm{C}$. Because the internal door surface is directly linked to the external surface, any change of the external surface temperature would increase or decrease the conductively heat transfer to the internal door. And the value for the dashboard and the seat is about $1.5^{\circ} \mathrm{C}$ and $2.5^{\circ} \mathrm{C}$ correspondingly. Indeed, the dashboard and the seats surface are mainly heated by the solar radiation. Only changing the external surface condition of the car body does not affect the solar illumination intensity on the internal surface. The effect of the external surface condition on the dashboard and the seats is mainly through the surface radiation heat transfer. The change of the surface temperature, for example the temperature at the door and the ceiling, will change the surface radiation heat transfer among internal surfaces. 


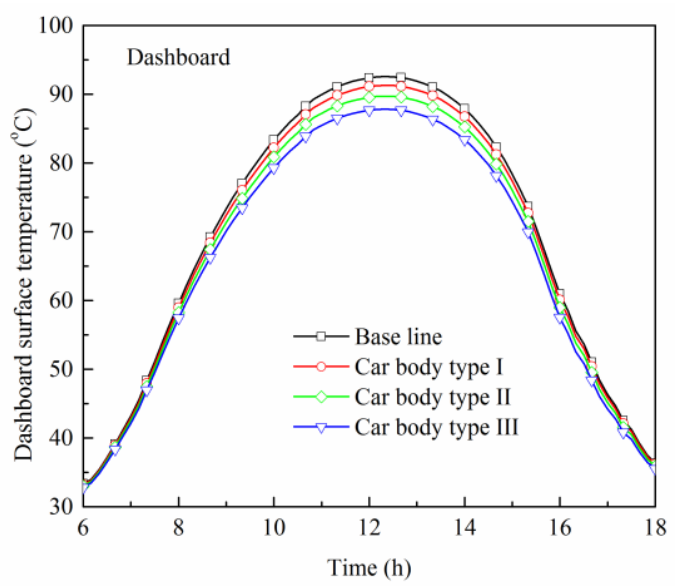

(a)

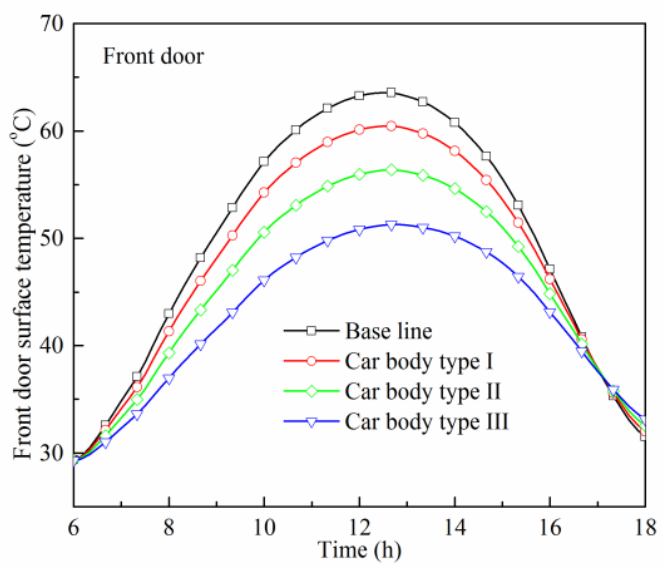

(c)

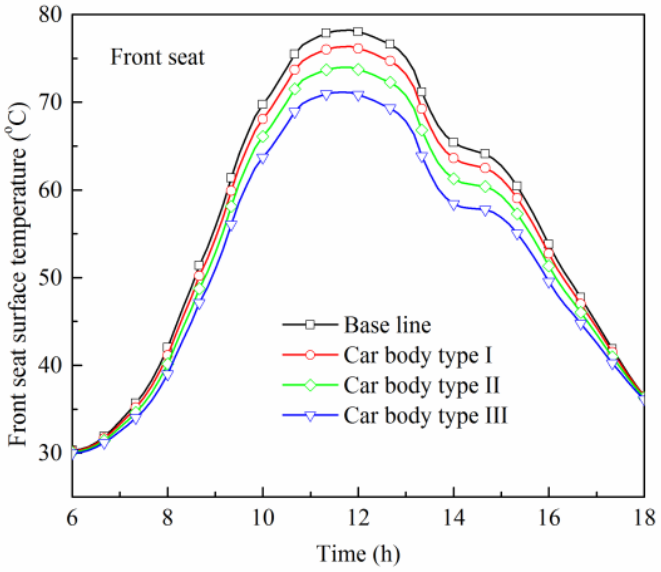

(b)

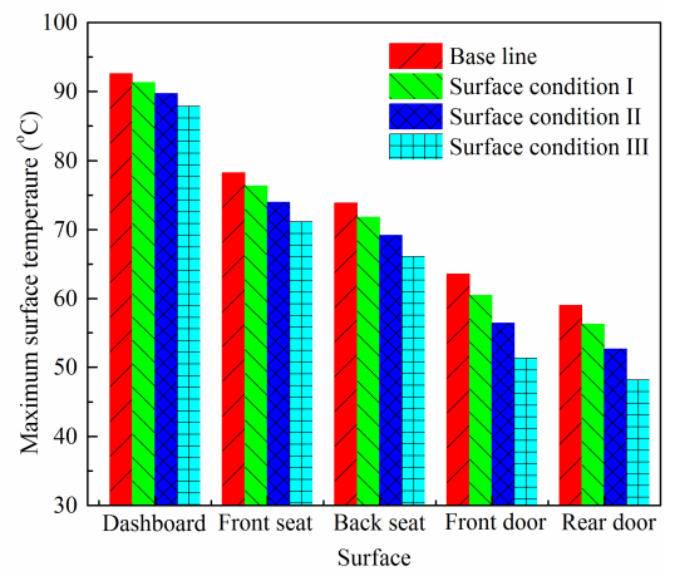

(d)

Figure 8. Effect of car body external surface conditions on the internal surface temperature

\section{Conclusions}

This study aims to find a way to obtain a more accurate surface thermal boundary conditions and decrease the internal surface temperature in a vehicle cabin in summer, then improve occupant's thermal comfort and reduce air conditioning power consumption. In this paper, we described the theoretical support of the numerical approach to study the thermal conditions in a vehicle cabin. Experimental and numerical investigations of temporal surface thermal conditions in summer conditions were carried out. The influence of the window glass and car body properties was quantificationally and systematically investigated. Based on the presented data and discussions, within the investigated parameter ranges, the following conclusions can be drawn:

(1) The surface temperature distribution inside a vehicle cabin is greatly depended on the solar radiation, and it is highly non-uniform through the cabin, the max temperature difference can exceed $30^{\circ} \mathrm{C}$. Moreover, there is a green house effect in the cabin, the interior air temperature is well above the outside ambient temperature under the sun.

(2) The numerical method presented in this paper could be reliably applied to the problem of surface thermal conditions inside a vehicle cabin under sun.

(3) The surfaces exposed to the sun, such as dashboard and the seats, are mainly heated by the solar radiation and dissipates by conductively, radiatively and convectively heat transfer. 
(4) The glass optical properties have a pronounced influence on the internal surface temperature, especially the surfaces exposed to the sun. With the transmittivity of window glass reducing every 0.2 , the maximum surface temperature reduces about $15{ }^{\circ} \mathrm{C}$ and $9{ }^{\circ} \mathrm{C}$ at the dashboard and seats correspondingly, but only $2^{\circ} \mathrm{C}$ on the door in this study.

(5) Increasing the reflectivity of the external surface can also results in a decrease of internal surface temperature. But, it's not so obvious as comparison to the window glass properties. With the absorptivity reducing by each 0.2 , the maximum temperature decrease about $1.5^{\circ} \mathrm{C}, 2.5^{\circ} \mathrm{C}$ and $4^{\circ} \mathrm{Con}$ the dashboard, seat and door correspondingly.

\section{Acknowledgements}

This project is supported by National Natural Science Foundation of China (Grant No. 51375170) and Guangdong province public monweal research and capacity building project (2014A020216052)

\section{References}

1. Y.D. Cheng, J.L. Niu, N.P. Gao, Thermal comfort models: A review and numerical investigation. Build Environ, 47:13-22(2012)

2. C. Walgama, S. Fackrell, M. Karimi, A. Fartaj, G.W Rankin, Passenger thermal comfort in vehicles-a review, P I Mecheng D-J Aut, 220: 543-562(2006)

3. J. Currle, Numerical simulation of the flow in a passenger compartment and evaluation of the thermal comfort of the occupants, SAE Paper No. 970529

4. C.H,Chien J.Y Jang, Y.H Chen, S.C Wu, 3-d numerical and experimental analysis for airflow within a passenger compartment, Int J Auto Tech-Kor, 9: 437-445 (2008)

5. H.J. Zhang, L. Dai, G.Q. Xu, al et, Studies of air-flow and temperature fields inside a passenger compartment for improving thermal comfort and saving energy, Part I: Test/numerical model and validation, Appl Thermeng, 29: 2022-2027(2009)

6. H.J. Zhang, L. Dai, G.Q. Xu, al et, Studies of air-flow and temperature fields inside a passenger compartment for improving thermal comfort and saving energy, Part II: Simulation results and discussion, Appl Thermeng, 29: 2028-2036(2009)

7. J. Rugh, V. Hovland, National and world fuel saving and $\mathrm{CO} 2$ emission reductions by increasing vehicle air conditioning COP, Proceedings from the 2003 Alternate Refrigerant Systems Symposium in Phoenix, AZ,( 2003)

8. R. McGuffin, R. Burke, C. Huizenga, H. Zhang, A. Vlahinos and G. Fu, Human thermal comfort model and manikin, SAE Paper 2002-01-1955

9. R. Levinson, H. Pan, G. Ban-Weiss, P. Rosado, R. Paolini, H. Akbari, Potential benefits of solar reflective car shells: Cooler cabins, fuel savings and emission reductions, Appl Energ. 88: 43434357(2011)

10. D. Turler, D. Hopkins, H. Goudey, Reducing vehicle ancillary loads using advanced thermal insulation and window technologies, SAE Paper No. 2003-01-1076.

11. P.B Hoke, C. Greiner, Vehicle paint radiation properties and affect on vehicle soak temperature, climate control system load, and fuel economy, SAE Paper No. 2005-01-1880 . 